\title{
Introducción a la Sección Especial
}

\section{Discriminación hacia Minorias Sexuales en Países Ibéricos y Latinoamericanos: Un Abordaje Psicosocial}

\author{
Introduction to the Special Section \\ Sexual Minorities' Discrimination in Iberian and \\ Latin American Countries: A Psychosocial Approach
}

\author{
Jaime Barrientos \\ Editor Invitado \\ Universidad Alberto Hurtado \\ Jorge Gato \\ Editor Invitado \\ Universidade do Porto
}

Hasta hace unas décadas atrás, la psicologia jugó un rol negativo respecto de la vida de lesbianas, gais, bisexuales y personas transgénero (LGBT). Sin embargo, la aparición de la noción de homofobia permitió poner el acento no en la homosexualidad, sino en las personas que ejercen violencia contra las personas LGBT (Barrientos Delgado, 2015). Paralelamente, en muchos países occidentales se han producido importantes avances legales a través de leyes antidiscriminación, de matrimonio igualitario o de identidad de género (Mendos, 2019). También, las actitudes hacia las personas LGBT han mejorado considerablemente en muchos países del mundo (Flores, 2019).

Este número especial presenta algunas investigaciones hechas desde la psicología sobre minorías sexuales en países ibéricos y latinoamericanos para contribuir a la superación de un déficit de investigación sobre esta temática. Esta sección especial de la revista Psykhe incluye siete estudios, dentro de los cuales se encuentran estudios empíricos y revisiones de literatura.

Las identidades transgénero han adquirido una relevancia en muchas sociedades occidentales. El primer artículo, de Barrientos, Saiz, Gómez, Guzmán-González, Espinoza-Tapia, Cárdenas y Bahamondes, presenta una revisión actualizada de la situación de las personas transgénero. Además, revisa la noción trans y cómo la psicología ha contribuido negativamente al patologizar dichas identidades trans. También, examina las investigaciones existentes sobre tales identidades desde una perspectiva psicosocial.

El segundo artículo, de Figueroa y Tasker, examina los discursos de las mujeres heterosexuales chilenas sobre la crianza de madres lesbianas y gais. Este es un tema muy actual en la discusión social y política de muchos países, persistiendo un fuerte debate sobre la homoparentalidad. Los resultados de este estudio muestran que la principal preocupación de las personas que participaron en el estudio fue que los niños de parejas del mismo género sean discriminados, debido a la identidad sexual de sus padres. Además, este estudio muestra que los niños en familias parentales gais o lesbianas todavía se consideran potencialmente vulnerables a prejuicios secundarios y, en el peor de los casos, en riesgo de interrupción de la identidad sexual y de género.

El artículo de Espinoza, Fernández, Riquelme e Irarrázaval describe el proceso de construcción de la identidad transgénero desde la perspectiva de los adolescentes. Este fue un estudio exploratorio realizado en Santiago, Chile. Entre los principales hallazgos se encontró que los adolescentes estudiados iniciaron muy tempranamente una trayectoria interesada por actividades del género no asignado al nacer y, a la vez, este interés era percibido con reticencia y/o rechazo de la familia y/o pares. Además, se encontró que este proceso va de la mano con sentimientos contradictorios que mueven hacia la búsqueda de información en el medio sociocultural para dar nombre y sentido a lo que les sucede. 
El artículo de Pereira de Souza, Costa y Strey, estudio realizado con jóvenes en Rio Grande do Sul, Brasil, investiga la violencia de género en las relaciones afectivas. Este interesante estudio describe la dificultad de definir diferentes modalidades de relaciones, así como el impacto del ideal de amor romántico en la invisibilidad de la violencia. El estudio muestra, además, la persistencia del imaginario del amor romántico, lo que limita las relaciones y la capacidad de percibir situaciones de violencia de género. Por último, el articulo describe cómo el ideal de amor romántico facilita el prejuicio con respecto a la orientación sexual.

El estudio de Longares, Rodríguez-Carballeira, Escartín y Garrido-Rosales, efectuado en España, analiza las experiencias de victimización y perpetración de abuso psicológico en relaciones de pareja intragénero, en las explicaciones de este abuso y en las dificultades experimentadas por las víctimas para identificarlo. Este es un fenómeno de importancia en la investigación psicosocial actual (Barrientos, Rodríguez-Carballeira, Escartín \& Longares, 2016). El estudio identifica estrategias de abuso psicológico, como el aislamiento de amistades, aislamiento en el hogar, control de la vida personal, abuso emocional, imposición del propio pensamiento y amenaza de outing a la pareja. Además, este estudio entrega algunas explicaciones para este abuso psicológico: influencia de la lesbofobia, bifobia y gayfobia interiorizada, concepción de la pareja como una posesión y vivencia previa de violencia.

El artículo de Posada Zapata y Agudelo Olarte reporta los hallazgos de un estudio efectuado en Medellín, Colombia, que buscó establecer el significado que tienen los hombres homosexuales sobre sus comportamientos de riesgo de contraer el VIH/SIDA. El estudio reporta que el carácter clandestino de los encuentros sexuales hace que el riesgo se asuma como una forma de vivir el afecto. También, el estudio describe una interesante asociación inversa entre el afecto y el riesgo asumido: a mayor presencia de afecto mayor evitación del riesgo, consecuente con la información sobre prevención, y a mayor ausencia de afecto mayor disposición al riesgo, buscando el reconocimiento y negando la información sobre protección.

Por último, en su artículo, Tomás Ojeda propone una aproximación crítica al lugar social y político de la psicoterapia en el debate actual sobre las ofertas de cambio de las sexualidades no heterosexuales. El artículo es una revisión cuidadosa, actualizada y crítica de dichas terapias y el lugar que estas tienen en el entramado psicoterapéutico contemporáneo, especialmente en Chile.

\section{Referencias}

Barrientos Delgado, J. (2015). Violencia homofóbica en América Latina y Chile. Santiago, Chile: El Buen Aire.

Barrientos, J., Rodríguez-Carballeira, A., Escartín, J. \& Longares, L. (2016). Violencia en parejas del mismo sexo: revisión y perspectivas actuales. Revista Argentina de Clínica Psicológica, 25, 289-298. Extraído de https://www.redalyc.org/pdf/2819/281948416008.pdf

Flores, A. R. (2019). Social acceptance of LGBT people in 174 countries, 1981 to 2017. Los Angeles, CA: University of California, Los Angeles, School of Law Williams Institute.

Mendos, L. R. (2019). Homofobia de Estado 2019. Genève, Suiza: Asociación Internacional de Lesbianas, Gays, Bisexuales, Trans e Intersex. Extraído de https://ilga.org/downloads/ILGA_Homofobia_de_Estado_2019.pdf 